$\Rightarrow$

\title{
An optical baton
}

\section{4}

Our results demonstrate that light can function as an optical baton for the highly tuneable and accurate manipulation of the levitated droplets

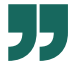

The smaller we go the more fascinating things become. For example, the properties of materials can change at the nanoscale and chemical reactivity might change when reactants are confined in microdroplets. A number of analytical techniques have made good use of the increased concentration that is achieved when an analyte is confined in a microdroplet. The precise manipulation of microdroplets is therefore a challenge that could lead to many opportunities within the field of microfluidics. Several approaches have been proposed for the levitation of microdroplets that usually make use of acoustic waves or applied magnetic fields. Most of these approaches also require complex machinery for the manipulation of the microdroplets. Now, writing in J. Phys. Chem. Lett., Rong Chen, Long Jiao and co-workers report

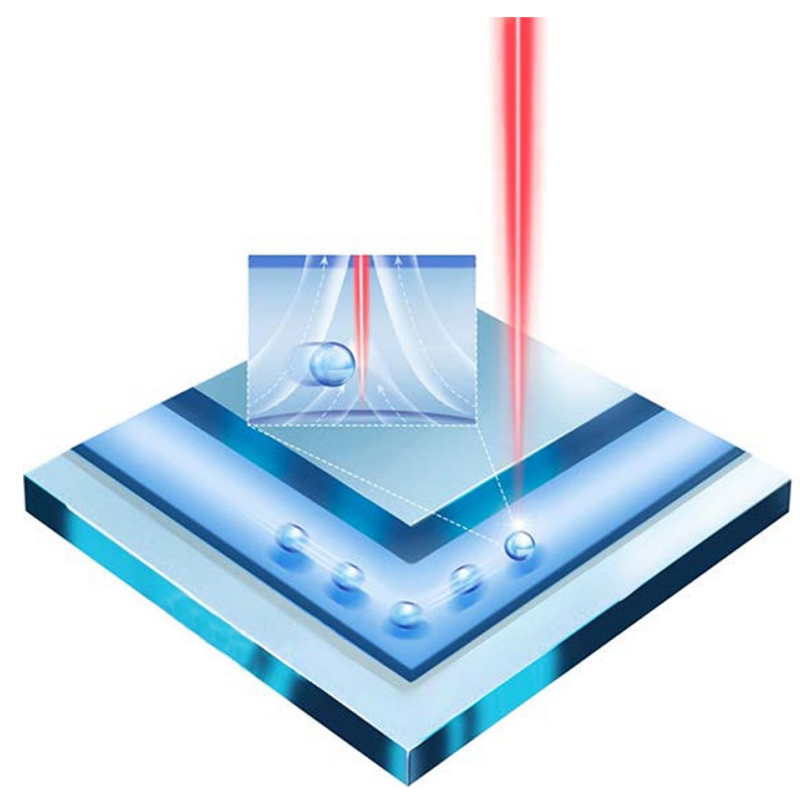

Credit: Adapted from Jiao, L. et al. (2019), ACS on a newly developed approach for the generation, manipulation and transportation of droplets that levitates them using light.

"Ultra-precise and highly flexible manipulation of microdroplets is very hard, even when various auxiliaries and lab chips are used," says Chen. "We have recently explored liquid photo-actuation for light-driven microfluidics. Light could serve as an efficient stimulus to actuate small amounts of liquid as it can provide distinct spatial and temporal resolution, which shows promising applications for the manipulation and analysis of bioassays, immunoassays, pharmaceuticals and materials."

The light-activated levitation approach proposed by the group at Chongqing University and Hong Kong Polytechnic University is surprisingly simple. A focused laser is used to heat a macrodroplet of water deposited on a hydrophilic surface. The heat generated by the laser induces a vaporization of water and the formation of microdroplets above the surface of the larger droplet. The microdroplets then flow across the surface of the macrodroplet with a velocity that depends on the contact angle between the water droplet and the surface - in other words, depending on the hydrophilicity or hydrophobicity of the solid surface. In the presence of a hydrophilic surface, the contact angle of the water droplet is small and the droplet surface is rather flat, inducing a slow vertical flow of the microdroplets. Once these levitate on top of the water surface, they tend to aggregate and grow in size until they start falling down on the water surface. However, evaporation flow from the interface pushes the microdroplets up. This balance between the gravitational forces and the vapour flow stabilizes the floating of the microdroplets and their size.

The group led by Chen was able to control the evaporation flow and size of the microdroplets $(10-40 \mu \mathrm{m})$ by changing the laser power and the curvature of the water surface. "We generated several micro droplets under high laser power and small water surface curvature but then adjusted the laser power and the water surface curvature to control their number and size," explains Chen. The trajectory of the formed floating microdroplets can also be precisely controlled. The team used the laser beam to move the floating microdroplets between different macrodroplets of water on a surface. They were able to precisely tune the speed, direction and position of the droplets by simply scanning the laser over the surface. Furthermore, the composition of the liquid does not seem to compromise the successful levitation of the microdroplets, as demonstrated in the case of 1,2-propanediol (1,2-PDO), 1,2-PDO/ $\mathrm{H}_{2} \mathrm{O}$ mixture and 1,2-PDO/vegetable glycerin mixture.

"We have demonstrated that creation, maintenance and manipulation of the levitated droplets can be achieved using a single light beam," remarks Chen. "Our results demonstrate that light can function as an optical baton for the highly tuneable and accurate manipulation of the levitated droplets, making this approach promising for future applications in complex materials transport and in situ reactions and analyses."

Gabriella Graziano

ORIGINAL ARTICLE Jiao, L. et al. Highly flexible and ultraprecise manipulation of light-levitated femtoliter/picoliter droplets. J. Phys. Chem. Lett. 10, 1068-1077 (2019) 